that $\lambda^{p^{2-1}}$ is the highest power of $\lambda$ contained in $D_{r}^{\prime}$. Hence

$$
D_{r}^{\prime}=c^{p-1} \lambda^{p^{2}-1},
$$

where $c$ is the product of the distinct prime factors of $m$ different from $p$.

From (1), (2), (3), (5), (7), (8), and (9) it then easily follows in both cases that

$$
N\left(D_{r}\right)=p^{p-2} \text {. }
$$

But in the field $k(\sqrt[p]{m})$ we have the following decomposition of $p$ into prime ideal factors, as was proved in the paper mentioned above :

$$
p=P^{p},
$$

if $b^{p-1}-a_{p-1}^{p-1}$ is not divisible by $p^{2}$, and

$$
p=P^{p-1} Q,
$$

if $b^{p-1}-a_{p-1}^{p-1}$ is divisible by $p^{2}$, where $P$ and $Q$ are different prime ideals of the first degree.

In the first case we obtain

$$
D_{r}=P^{p-2} .
$$

In the second case, however, our method does not enable us to determine the exact powers of $P$ and $Q$ which enter into $D_{r}$. We only know that

$$
D_{r}=P^{x} \cdot Q^{y}
$$

where $x+y=p-2$.

PuRdue University, October, 1910.

\title{
NOTE ON RECIPROCAL FIGURES IN SPACE.
}

BY PROFESSOR PETER FIELD.

Maxwell [Collected Works, page 523. Also see Rankine, Philosophical Magazine for February, 1864] defines figures in three dimensions as reciprocal when they can be so placed that every line in the one is perpendicular to a plane face of the other and every vertex in the one is represented by a closed polyhedron with plane faces in the other.

The simplest case [Maxwell, loc. cit., page 524] is that of 5 
points in space with their 10 connecting lines. This forms a figure composed of 5 tetrahedrons and having 10 triangular faces. The 5 points which are the centers of the spheres circumscribing the 5 tetrahedrons, when connected, form another figure which is reciprocal to the first, and stresses proportional to the areas of the faces of one figure when applied along the edges of the other will keep its vertices in equilibrium. In either one of these figures, 4 of the points might be considered as the vertices of a tetrahedral frame and the fifth point as the point of concurrence of 4 forces which keep the frame in equilibrium. The reciprocal figure then exhibits the magnitudes of the forces and the stresses in the different members of the frame.

Although the idea of reciprocal figures in space is not of much importance in mechanics, because a reciprocal figure can be constructed only in case certain conditions are satisfied, nevertheless it seems of some interest to construct a figure which can be interpreted as representing a frame held in equi-

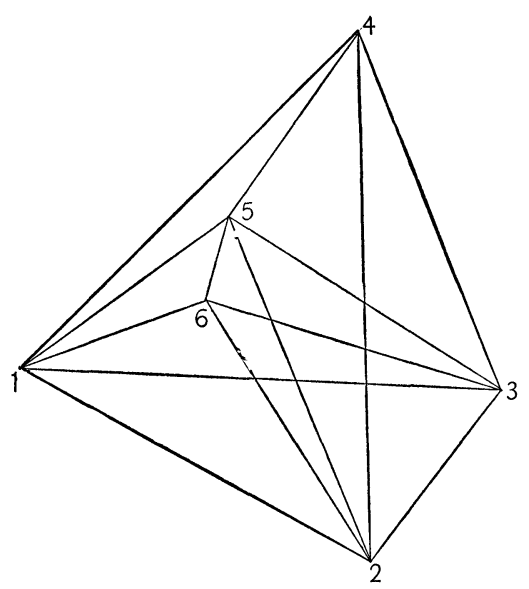
librium by four nonconcurrent forces and for which a reciprocal figure can be constructed.

In the figure at the left, $1,2,3,4$ represents a given frame. The line of action of the force at 4 is taken as 45,5 being any point on the line. As the forces acting at the 4 vertices are in equilibrium, they lie on a hyperboloid [Föppl, Technische Mechanik, volume II, page 158] and it is therefore possible to draw a line through 5 which meets the lines of action of the three remaining forces. Let 6 be any point on this line and join the points as in the figure. The stresses in 15 and 16 can be chosen arbitrarily (as the whole figure regarded as a frame has two more edges than are necessary to make it rigid) and the remaining stresses are then fixed.

The figure can be considered as made up of the tetrahedrons 
$1234,1236,1256,1356,2356,1254,1354$, and 2354 . About each of these tetrahedrons a sphere can be circumscribed and the centers of these spheres could be taken as the vertices of a figure which is reciprocal to the given one, i. e., it would be a possible solution. The most general reciprocal figure which can be constructed must contain arbitrary constants corresponding to the fact that the stresses along 15 and 16 can be chosen arbitrarily. This is brought out clearly by constructing the reciprocal figure by the method used by Klein and Wieghardt (Archiv der Mathematik und Physik, 3 Reihe, Band 8, Heft 2) which may be summarized as follows:

There are given two sets of rectangular axes that are parallel. Assume a system of cells in the $x, y, z$ space which are so arranged that they fill a certain boundary polyhedron doubly. Assign to each cell $C_{j}$ a linear function

$$
t+\theta_{j}=\xi_{j} x+\eta_{j} y+\zeta_{j} z
$$

To the cell $C_{j}$ in the $x, y, z$ space there corresponds a point $P_{j} \equiv\left(\xi_{j}, \eta_{j}, \zeta_{j}, \theta_{j}\right)$ whose projection in the $\xi, \eta, \zeta$ space has the coordinates $\xi_{j}, \eta_{j}, \zeta_{j}$. Similarly, to the cell $C_{l_{k}}$ there corresponds the point $P_{k^{\circ}}$ If $C_{k}$ and $C_{j}$ are neighboring cells, the plane between the two has the equation

$$
\left(\xi_{j}-\xi_{k}\right) x+\left(\eta_{j}-\eta_{k}\right) y+\left(\zeta_{j}-\zeta_{k}\right) z-\left(\theta_{j}-\theta_{k}\right)=0,
$$

which is a plane perpendicular to the line $P_{j} P_{k}$. If the origin is taken as a vertex in the reciprocal diagram, $t=0$ for the corresponding cell. From the equations of the boundary planes it is possible to find the values of $t$ (and consequently the coordinates of the vertices in the reciprocal diagram) for the other cells. In this way the reciprocal figure can be constructed.

In order to prevent the detail work from becoming too cumbersome, take the frame as $P_{1} \equiv(0,0,0), P_{2} \equiv(1,0,0)$, $P_{3} \equiv(0,1,0), P_{4} \equiv(0,0,1), P_{5} \equiv(1,1,1)$, and $P_{6} \equiv(1,-2,1)$. The relation between the figure and its reciprocal can then be tabulated as follows: 


$\begin{array}{cccccc}\text { Cell } & \text { Value of } t & \xi & \eta & \zeta & \theta \\ 1234 & 0 & 0 & 0 & 0 & 0 \\ 5123 & b z & 0 & 0 & b & 0 \\ 5134 & b x & b & 0 & 0 & 0 \\ 5124 & b y & 0 & b & 0 & 0 \\ 6235 & c(x+y)+(b-c) z-c & c & c & b-c & c \\ 6345 & (b-c) x+c(y+z-1) & b-c & c & c & c \\ 6234 & (x+y+z-1)(3 c-b) & 3 c-b 3 c-b 3 c-b 3 c-b \\ 6245 & (b-c)(x+z-1)+c y & b-c & c & b-c b-c \\ \text { UNIVRRSITY OF MICHIGAN } & & & & \end{array}$

\section{MATHEMATICAL PHYSICS FOR ENGINEERS.}

Einführung in die Theorie des Magnetismus. Von R. Gass. Leipzig, Teubner, 1908. vi $+110 \mathrm{pp}$.

Einführung in die Maxwellsche Theorie der Elektrizität und des Magnetismus. Von Cl. Schaefer. Leipzig, Teubner, 1908. viii $+174 \mathrm{pp}$.

Funktionentafeln mit Formeln und Kurven. Von E. JaHNKE und F. Emde. Leipzig, Teubner, 1909 . xii +176 pp.

Professor Jahnke is editing a series of texts for engineers. The exact title of the series is Mathematisch-physikalische Schriften für Ingenieure und Studierende. It is published by the firm of B. G. Teubner with the usual typographical excellence of all such works issued by this house. The titles of the volumes which have already appeared, in addition to the three at the head of this review, are Elektromagnetische Ausgleichsvorgänge in Freileitungen und Kabeln, Die Theorie der Besselschen Funktionen,* Die Vektoranalysis und ihre Anwendungen in der theoretischen Physik (2 volumes), $\uparrow$ Theorie der Kräftepläne.\$ There are further announced some twentyfive volumes to which titles and authors have already been assigned, and we are assured that a longer continuation of the series is in contemplation. The texts, or should they be called tracts, are all of moderate size, varying from a bit over 100 to somewhat less than 200 pages. From those which have hitherto appeared it is already evident that each has a definite aim and mission to fulfil, which indeed it does fulfil, and that the series as a whole, if not carried so far as to lose its vitality, will form a

* Reviewed in this Bulletin, volume 16 (1910), p. 385.

+ Reviewed in this Bulletin, volume 17 (1910), p. 100.

$\ddagger$ Still others have appeared since the date of this review. 